\title{
Effect of rehabilitation on a patient suffering from a tuberculous brain abscess with Gerstmann's syndrome: case report
}

This article was published in the following Dove Press journal:

Neuropsychiatric Disease and Treatment

3 May 2012

Number of times this article has been viewed

\author{
Chih-Lan Kuo' \\ Sui-Foon Lo',2 \\ Chun-Lin $\mathrm{Liu}^{3}$ \\ Chia-Hui Chou ${ }^{4}$ \\ Li-Wei Chou ${ }^{1,2,5}$ \\ 'Department of Physical Medicine \\ and Rehabilitation, China Medical \\ University Hospital, Taichung, \\ Taiwan; ${ }^{2}$ School of Chinese Medicine, \\ College of Chinese Medicine, China \\ Medical University, Taichung, Taiwan; \\ ${ }^{3}$ Department of Neurosurgery, \\ China Medical University Hospital, \\ Taichung, Taiwan; ${ }^{4}$ Department of \\ Infectious disease, China Medical \\ University Hospital, Taichung, Taiwan; \\ ${ }^{5}$ Department of Physical Therapy, \\ China Medical University, Taichung, \\ Taiwan
}

\begin{abstract}
There are few reports in the literature of tuberculous brain abscess. Tuberculous brain abscess usually occurs in an immunocompromised host. Almost all previously documented cases have involved acquired immune deficiency syndrome. We encountered a 53-year-old right-handed immunocompetent male who was initially suspected of having a cerebrovascular accident due to acute-onset right hemiparesis and paresthesia. A tentative diagnosis of brain tumor versus brain abscess was made on imaging studies. The patient was finally diagnosed with a tuberculous brain abscess based upon deterioration on imaging and a positive tuberculosis culture. The tuberculous brain abscess was located in the left parietal lobe, which resulted in Gerstmann's syndrome and right-sided apraxia. Stereotactic surgery was performed. He was also given antituberculosis chemotherapy and comprehensive rehabilitation. Considerable improvement was noted after rehabilitation. The patient even returned to a normal life and work. Our case demonstrates that an aggressive intensive inpatient rehabilitation program combined with stereotactic surgery and effective antituberculosis therapy play an important role in improving the outcome for patients with tuberculous brain abscess, Gerstmann's syndrome, and right-sided apraxia.
\end{abstract}

Keywords: tuberculous brain abscess, Gerstmann's syndrome, rehabilitation

\section{Introduction}

Tuberculosis (TB) most commonly affects the lungs, ${ }^{1}$ with extrapulmonary TB observed in only $20 \%$ of all cases. ${ }^{2} \mathrm{~TB}$ of the central nervous system accounts for approximately $1 \%$ of all TB cases. ${ }^{3}$ Most cases of TB affecting the central nervous system develop in immunocompromised persons with pulmonary $\mathrm{TB},{ }^{4,5}$ and commonly manifests as meningitis or tuberculoma ${ }^{6,7}$ Tuberculous brain abscess is a very uncommon form of TB in the central nervous system. ${ }^{6,7}$

Diagnosis of a tuberculous brain abscess may be very difficult in a person without a history of contact with TB or without pulmonary TB. Symptoms of tuberculous brain abscess also depend on the location of the lesion. A tuberculous brain abscess may mimic a cerebrovascular accident or a brain tumor. Lesions located in the left parietal lobe may result in Gerstmann's syndrome. Here we present a case presenting as Gerstmann's syndrome secondary to a rare tuberculous brain abscess without primary pulmonary lesions or immunocompromise.

Department of Physical Medicine and Rehabilitation, China Medical University Hospital, No 2 Yuh-Der

Road, Taichung 404, Taiwan

Tel +88 6422052 I 2 I ext 238I

Fax +88 642202604 I

Email chouliwe@tcts.seed.net.tw

\section{Case report}

A 53-year-old right-handed man complained of acute-onset right-sided weakness and paresthesia. He was reportedly healthy with no chronic or infectious diseases. 
The patient could not control the movement of his right-sided limbs very well. Loss of proprioception in the right limbs was also noted. His vital signs were stable and he had no fever. General physical examination was normal. However, neurologic examination revealed left-sided and right-sided confusion (difficulty in distinguishing the directions right and left), right-sided limb apraxia, impairment of light touch, impaired pinprick sensation and proprioception on the right side, as well as dyscalculia and dysgraphia. Muscle strength on the right side was grade 3 out of 5 on the Medical Research Council scale (indicating that active movement against gravity is possible). ${ }^{8}$ The initial impression was of a cerebrovascular accident because of the acute onset and focal deficits. There were no obvious risk factors for stroke, nor any symptoms or signs of increased intracranial pressure.

Hematologic investigations and chest x-ray were normal. Cranial computed tomography (CT) showed a brain mass with edema of the peripheral white matter in the left parietal lobe (Figure 1A). Magnetic resonance imaging of the brain revealed a mass in the left parietal lobe with irregular central necrosis and edema of the peripheral white matter (Figure 1B). A metastatic tumor or brain abscess was suspected. Alpha-fetoprotein, carcinoembryonic antigen, cancer antigen 15-3, prostate-specific antigen, cancer antigen 125 , and carbohydrate antigen 19-9 were all within normal limits. Other laboratory examinations, including aspartate transaminase, alanine aminotransferase, gamma-glutamyl transferase, bilirubin, and alkaline phosphatase, were normal. Intravenous dexamethasone $4 \mathrm{mg}$ every eight hours was prescribed in the emergency room due to the severe brain edema found on CT.

The patient's neurologic status deteriorated into right hemiplegia two days after admission. His muscle strength on the right side deteriorated from grade 3 to 0 out of 5 . A subsequent cranial CT scan showed that the brain mass had increased in size, with ring-like enhancement and more severe edema of the peripheral white matter (Figure 1C). The findings favored a brain abscess rather than a metastatic lesion. Stereotactic surgery was performed to obtain a tissue diagnosis and decompression of the mass effect. During the operation, approximately $6 \mathrm{~mL}$ of pus was aspirated. The pathology report showed predominantly neutrophils with necrotic tissue and no caseation or granuloma tissue, which was compatible with a brain abscess (Figure 2A). ZiehlNeelsen staining of the pus revealed that acid-fast bacilli were present (Figure 2B). Serial cultures of pus from the abscess were conducted, which showed the Mycobacterium tuberculosis complex. Microbiologic analysis demonstrated sensitivity to ethambutol, pyrazinamide, rifampin, and isoniazid. Human immunodeficiency virus serology was negative.

Pulmonary tuberculosis in this patient was unlikely due to the absence of cough or other respiratory symptoms during his clinical course. The initial and follow-up chest x-ray at 12 months were unremarkable, so we did not perform a CT chest after consultation with a chest specialist. Instead of performing a conventional sputum culture, we arranged a sputum polymerase chain reaction examination which was negative.

The criteria for diagnosis of a tuberculous brain abscess include presence of a brain abscess and acid-fast bacilli in pus and absence of caseation and formation of granuloma. ${ }^{8}$ This case met all these criteria, so was diagnosed as a primary tuberculous brain abscess. The patient received antituberculous chemotherapy consisting of oral ethambutol $1200 \mathrm{mg} /$ day, pyrazinamide $1500 \mathrm{mg} /$ day, rifampin $600 \mathrm{mg} /$ day, and isoniazid $300 \mathrm{mg} /$ day. His muscle strength
A

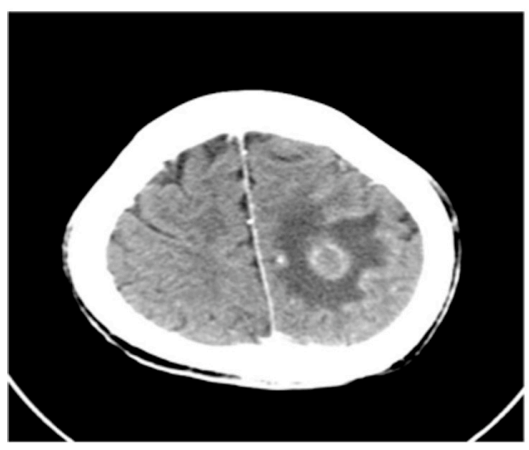

B

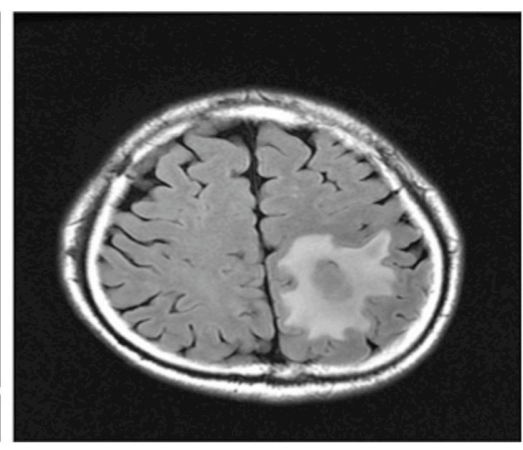

C

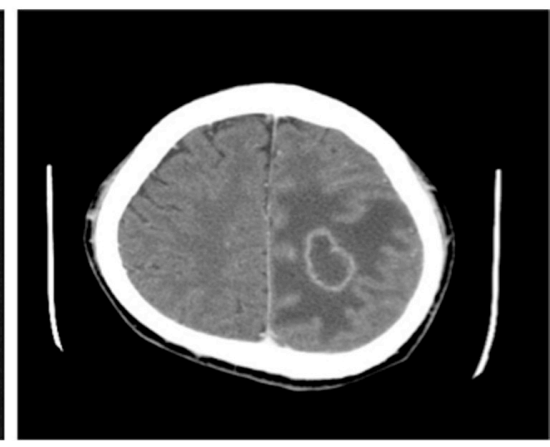

Figure I (A) Computed tomography scan at admission showing a brain mass with edema of the peripheral white matter in the left parietal lobe. (B) Brain MRI T2-flair revealed a mass in the left parietal lobe measuring approximately $23 \mathrm{~mm} \times 17 \mathrm{~mm}$, with irregular central necrosis and edema of the peripheral white matter. (C) Brain computed tomography scan obtained two days after admission showing a mass in the left parietal lobe, approximately $32 \mathrm{~mm} \times 22 \mathrm{~mm}$, with central cystic change, ring-like enhancement, and edema of the peripheral white matter. 

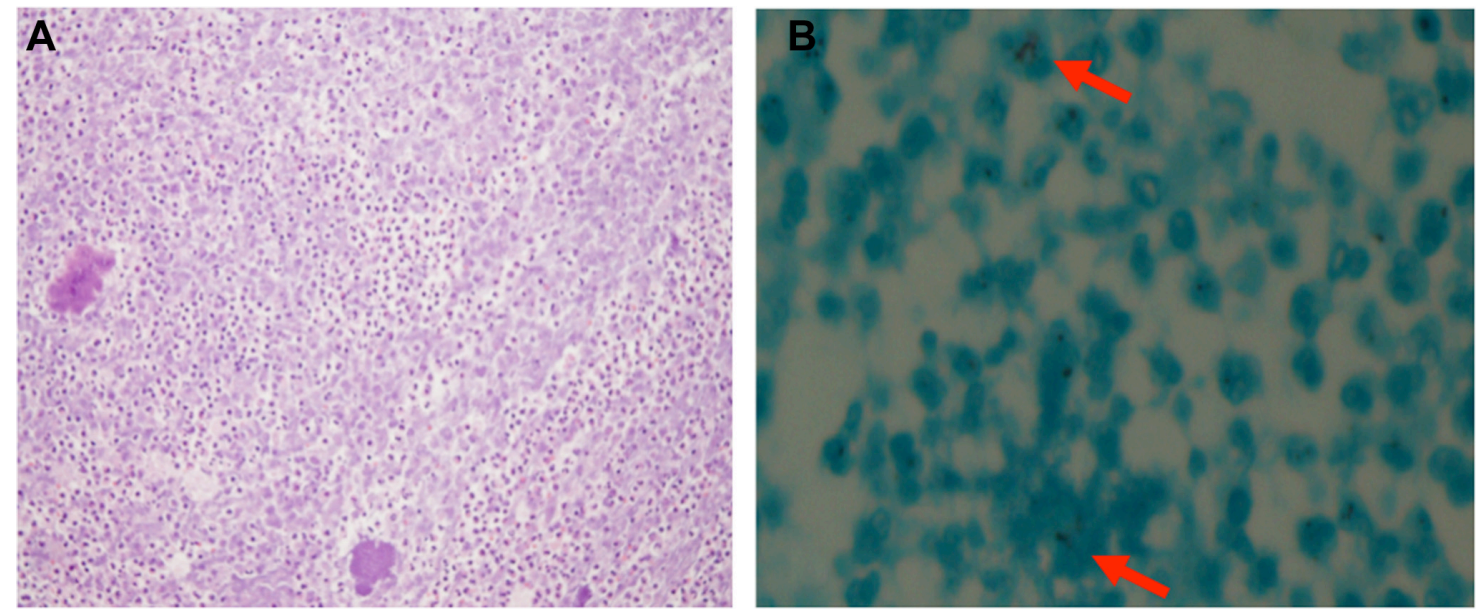

Figure 2 (A) hematoxylin and eosin stain showing necrotic tissue with neutrophilic infiltration (original magnification $\times 400$ ). (B) Ziehl-Neelsen stain demonstrating acid-fast bacilli (original magnification $\times 400$ ).

on the right side gradually recovered (from grade 3 to 0 out of 5) after aspiration of the abscess and antituberculous chemotherapy. Dexamethasone was continued during admission and then changed to an oral formulation after one month. Dexamethasone was suspected to be helpful for the cerebral edema causing his focal neurologic signs.

The patient was transferred to our physical medicine and rehabilitation ward for further rehabilitation. Initially, upon arrival for rehabilitation, he could only walk with the use of a walker. Severe left-right confusion, right-sided apraxia, dyscalculia, and dysgraphia were noted. He needed moderate assistance in most activities of daily living. His functional independence measure score was 65 . The patient was diagnosed as having a tuberculous brain abscess in the left parietal lobe with Gerstmann's syndrome and right-sided apraxia. Physical therapy, occupational therapy, and speech therapy were arranged. In addition, to regain somatic sensation and motor control of the right side of his body, training was focused on the right-left confusion and apraxia. The sensory rehabilitation program addressed various perception deficits and impairment of hand-eye coordination. The motor training program included limb and trunk motion, posture, and balance training, which finally progressed to ambulation training. Speech therapy included writing and cognitive training. After antituberculous chemotherapy and rehabilitation, the patient was able to walk without assistance and had almost recovered normalcy at four months. His left-right confusion, right-sided apraxia, dyscalculia, and dysgraphia improved significantly.

He continued to receive antituberculous chemotherapy for a total of 18 months. We repeated the chest $\mathrm{x}$-ray several times over the course of 12 months and all were normal. Subsequent magnetic resonance imaging showed that the previous brain abscess had almost completely resolved.

\section{Discussion}

The pathogens commonly found in brain abscesses are Staphylococcus aureus, Streptococcus viridans, anaerobes, and Gram-negative bacilli. ${ }^{9}$ M. tuberculosis is a rare pathogen in brain abscesses and is often seen in immunocompromised hosts. With the advent of acquired immune deficiency syndrome, more cases have been diagnosed, but very few have been reported in immunocompetent patients negative for human immunodeficiency virus.

A previous case report and a review of the treatment of tuberculous brain abscess have shown that a combination of microsurgery and antituberculous chemotherapy seemed to be a better treatment method. ${ }^{11,12}$ There has been no report on how rehabilitation programs affect the prognosis or whether rehabilitation programs are beneficial to patients with tuberculous brain abscess who have already received surgery and antituberculous chemotherapy.

Gerstmann's syndrome is characterized by dyscalculia or acalculia (impaired ability or inability to calculate), dysgraphia or agraphia (impaired or inability to write), finger anomia (inability to name individual fingers, such as the index finger or thumb), and right-left confusion (inability to distinguish which side is right or left). ${ }^{13}$ Gerstmann's syndrome is associated with lesions or dysfunction of the left angular gyrus in the parietal lobe. ${ }^{14}$ Our patient had a tuberculous brain abscess in the left parietal lobe. This condition led to Gerstmann's syndrome and right-sided apraxia. Gerstmann's 
syndrome is a disease that is difficult to diagnose because it is usually associated with aphasia. ${ }^{15}$ Comprehension and fluency of speech are usually impaired. Our patient did not have aphasia, so was able to express the typical characteristics of Gerstmann's syndrome. Thus, he was easily diagnosed.

We closely observed the patient and followed his condition after rehabilitation. His right-sided apraxia and right-left confusion improved significantly. His functional status and functional independence measure score also improved significantly. Supervision was needed for most activities of daily living and the functional independence measure increased from 65 to 110 . He returned to normal life and work after four months of rehabilitation. He had a better prognosis and faster recovery in comparison with similar cases reported in the literature. Aggressive and intensive inpatient rehabilitation programs combined with stereotactic surgery and effective antituberculous therapy after an early diagnosis may result in a better outcome.

\section{Conclusion}

This report describes a rare case of an immunocompetent person suffering from a tuberculous brain abscess with subsequent Gerstmann's syndrome and right-sided apraxia. The patient recovered and returned to normal life after antituberculous chemotherapy and rehabilitation. This case demonstrates that aggressive and intensive inpatient rehabilitation programs combined with stereotactic surgery and effective antituberculous therapy play an important role in the management of this condition.

\section{Disclosure}

The authors report no conflicts of interest in this work.

\section{References}

1. Frieden TR, Sterling TR, Munsiff SS, et al. Tuberculosis. Lancet. 2003;362(9387):887-899.

2. Oncul O, Baylan O, Mutlu H, et al. Tuberculous meningitis with multiple intracranial tuberculomas mimicking neurocysticercosis clinical and radiological findings. Jpn J Infect Dis. 2005;58(6):387-389.

3. Rock RB, Olin M, Baker CA, et al. Central nervous system tuberculosis: pathogenesis and clinical aspects. Clin Microbiol Rev. 2008;21(2): 243-261.

4. Farrar DJ, Flanigan TP, Gordon NM, et al. Tuberculous brain abscess in a patient with HIV infection: case report and review. Am J Med. 1997; 102(3):297-301.

5. Velasco-Martínez JJ, Guerrero-Espejo A, Gómez-Mampaso E, et al. Tuberculous brain abscess should be considered in HIV/AIDS patients. AIDS. 1995;9(10):1197-1199.

6. Lesprit P, Zagdanski AM, de la Blanchardière A, et al. Cerebral tuberculosis in patients with acquired immunodeficiency syndrome (AIDS). Report of 6 cases and review. Medicine. 1997;76(6):423-431.

7. Whitener DR. Tuberculous brain abscess. Report of a case and review of the literature. Arch Neurol.1978;35(3):148-155.

8. Medical Research Council. Aids to Examination of the Peripheral Nervous System. Memorandum 45. London, UK: Her Majesty's Stationary Office; 1976.

9. Ersoy Y, Ates O, Onal C, et al. Cerebellar abscess and syringomyelia due to isoniazid-resistant mycobacterium tuberculosis. J Clin Neurosci. 2007;14(1):86-89.

10. Calfee DP, Wispeley B. Brain abscess. Semin Neurol. 2000;20(3): 353-360.

11. Gazzaz M, Bonyaakoub FA, Elkhamlichi A. Tuberculous cerebellar abscess. Acta Neurol Belg. 2000;100(1):146-147.

12. Ramesh VG, Sundar KS. Concomittant tuberculosis and pyogenic cerebellar abscess in a patient with pulmonary tuberculosis. Neurol India. 2008;56(1):91-92.

13. Mayer E, Martory MD, Pegna AJ, et al. A pure case of Gerstmann syndrome with a subangular lesion. Brain. 1999;122(Pt 6):1107-1120.

14. Carota A, Di Pietro M, Ptak R, et al. Defective spatial imagery with pure Gerstmann's syndrome. Eur Neurol. 2004;52(1):1-6.

15. Rusconi E, Pinel P, Dehaene S, et al. The enigma of Gerstmann's syndrome revisited: a telling tale of the vicissitudes of neuropsychology. Brain. 2010;133(Pt 2):320-332.
Neuropsychiatric Disease and Treatment

\section{Publish your work in this journal}

Neuropsychiatric Disease and Treatment is an international, peerreviewed journal of clinical therapeutics and pharmacology focusing on concise rapid reporting of clinical or pre-clinical studies on a range of neuropsychiatric and neurological disorders. This journal is indexed on PubMed Central, the 'PsycINFO' database and CAS.

\section{Dovepress}

The manuscript management system is completely online and includes a very quick and fair peer-review system, which is all easy to use. Visit http://www.dovepress.com/testimonials.php to read real quotes from published authors. 